\title{
CRYPTIC DIVERSITY IN INTESTINAL PROTISTS
}

\author{
CLARK C.G.*
}

\section{Summary:}

In the past few years our understanding of genetic variation within and between species of intestinal parasitic protists has changed significantly. New species names have been assigned and others have been dropped in response to new data. In this review, I summarise these findings and discuss their implications for future studies. In several cases the findings suggest that caution needs to be exercised to prevent premature conclusions being reached.

KEY WORDS : Blastocystis, Dientamoeba, Entamoeba, Cryptosporidium, ribosomal DNA.

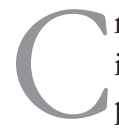
ryptic genetic diversity has been in the headlines in recent times. Most of the attention has been paid to discoveries made through the efforts to "barcode" animals. This has led to the discovery of cryptic species in many groups, from birds to insects. Barcoding in this context consists of PCR amplification of a portion of the mitochondrial genome of an organism, generation of a single pass sequence from the product, and database or phylogenetic comparisons using the sequence data obtained (Hebert et al., 2003). While the identification of cryptic species has generated excitement and surprise among the zoological fraternity, it will have come as no surprise to any of us working on unicellular parasites, especially those working on organisms with "limited" morphology, where such discoveries have been ongoing for a number of years. Several years ago I reviewed the status of cryptic diversity in intestinal protists (Clark, 2000). Since that time a number of additional findings have been made that deserve wider attention as they have implications beyond the limits of the genera they represent. Starting in the 1970s, all studies on Dientamoeba fragilis used the isolate $\mathrm{Bi} / \mathrm{PA}$ which was cultured by Balamuth from the faeces of a symptomatic female in $1940 \mathrm{~s}$ (Balamuth, 1946). This isolate was used for antigenicity studies (Dwyer, 1972a, 1972b, 1974) and antibody production (Chan et al., 1993) and later the small subunit ribosomal RNA gene (SSU-rDNA) was sequenced (Sil-

\footnotetext{
* Infectious and Tropical Disease, London School of Hygiene and Tropical Medicine, Keppel Street, London WC1E 7HT, GB.

Tel.: 44-20-7927-2351 - Fax: 44-20-7636-8739.

E-mail: graham.clark@1shtm.ac.uk
}

berman et al., 1996). In part this focus on one isolate was because it was available from the American Type Culture Collection and in part it was because many researchers found it difficult to establish new strains in culture. In 2000, it was shown that two genetic variants of $D$. fragilis exist in humans (Johnson \& Clark, 2000) and subsequent sequencing has shown the SSUrDNA divergence to be substantial (ca $4 \%$ ) (Stark et al., 2005a). Subsequently, several groups have used PCR and either restriction fragment length polymorphism or sequencing to study the distribution and diversity of D. fragilis across the world. What is most remarkable is that with one exception, all infections studied since 2000 have involved the "new" genotype (Johnson \& Clark, 2000; Peek et al., 2004; Windsor et al., 2004; Stark et al., 2005a, 2005b). In other words, Bi/PA is not representative of the majority of $D$. fragilis circulating in the human population. Is this observation important? Perhaps not, but it does mean that the conclusions of all studies performed on $\mathrm{Bi} / \mathrm{PA}$ must be viewed with some caution as the results may not always apply to the more common $D$. fragilis genotype.

Fortunately, the abundance of the "new" genotype of $D$. fragilis meant that its existence was quickly identified and researchers developing tools for DNA-based diagnosis were able to incorporate this information into their methodology. If the reverse had been true, however, it is possible that the existence of a divergent, minor variant might have been missed. This could have happened in the case of a recently uncovered E. histolytica-like organism.

The existence of two distinct organisms within what was being called E. histolytica based on morphology was recognised almost 30 years ago (Sargeaunt et al., 1978) and the recognition of E. histolytica and E. dispar as distinct species was given WHO approval in 1997 (Anonymous, 1997a, 1997b). Many DNA-based tools for the differentiation of E. histolytica and E. dispar have been developed and tested over the years and their specificity has been unquestioned as has the uniformity and integrity of the two species. This view has been challenged by two recent papers describing an amoeba from macaques that is virulent in animal models (Tachibana et al., 2007; Takano et al., 2007). Both groups 
used sequencing of several genes to show that this amoeba is specifically related to but distinct from $E$. histolytica. The SSU-rDNA differs from that of E. histolytica by $0.8 \%$, almost $50 \%$ of the divergence between E. histolytica and E. dispar. Analysis of all other genes studied to date shows the same result - these amoebae fall outside the range of variation encompassed by E. histolytica from humans. One group has proposed that this amoeba is a "subspecies" of E. histolytica (Takano et al., 2007) while the other has proposed that it should be accorded species status and proposed revival of the name E. nuttalli (Tachibana et al., 2007). I tend to agree with the latter.

Why is this finding of concern? It illustrates the potential for important variation being overlooked if diagnosis is based on PCR amplification alone. In the case of $E$. nuttalli, every pair of PCR primers examined that were thought to be specific for E. histolytica would also amplify a homologous product from this new amoeba. Without sequencing we would never have suspected that these isolates represented a new organism and they would have simply been identified as E. histolytica. In this case it seems that the new species does not normally infect humans. Extensive isoenzyme analyses of human isolates performed in the 1970s and 1980s would have identified any $E$. nuttalli infections had they been found, as the genetic divergence between the hexokinase and glucose phosphate isomerase genes of E. histolytica and E. nuttalli would be predicted to result in significant migration differences (Tachibana et al., 2007).

Traditionally, classification of Entamoeba species has been based on cyst morphology and host. For the most part, molecular data have upheld these identifications despite the paucity of morphological variation and uncertainty of host specificity in this genus. There have been, however, some exceptions. A survey of intestinal parasitic protists in Hué, Vietnam (Blessmann et al., 2002), found a large percentage of individuals to be infected with a uninucleated cyst-producing Entamoeba. Such infections are generally regarded as rare in humans so domestic animals in the same region were screened to try and identify a zoonotic source. Local pigs were also found to be infected with a uninucleated cyst producer (Clark et al., 2006). Based on the host and the cyst such organisms would traditionally be assigned to the species E. polecki. However, SSU-rDNA sequencing showed the pig amoeba to be only distantly related to both previously studied E. polecki isolates and the human amoeba from Hué. Indeed it proved to be specifically related to E. gingivalis, a non-cyst-producing inhabitant of the human mouth (Clark et al., 2006). While this finding may tell us something about the evolutionary history of E. gingivalis, it also illustrates that traditional criteria for species identification by microscopy can be very misleading and result in important diversity being overlooked. The pig amoeba has been assigned the pre-existing name E. suis which had fallen into disuse as a synonym for $E$. polecki.

Entamoeba also provides an illustration of a dilemma facing researchers working on a variety of parasite groups - when does a genetic difference become a species difference? In organisms that appear to be largely if not exclusively asexual this is a somewhat philosophical question. In my opinion the decision to assign new names to organisms should be a question of utility and clarity. The decision to revive the name E. dispar (Diamond \& Clark, 1993) was justified on the basis of differentiating a non-invasive parasite from one that can cause serious disease and death. Isolates of the two species formed discrete and consistent clades and they could be distinguished easily using any number of markers. The same situation does not always apply. In humans, four distinct types of uninucleated cyst-producing Entamoeba infections have been identified (Verweij et al., 2001). One of these has also been found in monkeys, a second has been found in pigs, and a third has been found in both pigs and ostriches (Clark et al., 2006). Traditionally, based on the host species, the first type would have been called E. chattoni and the second $E$. polecki, while the third has been described as E. struthionis (Ponce Gordo et al., 2004 ), despite having been previously found in humans. These organisms are genetically very closely related, although more divergent than E. histolytica and E. dispar. None appear to be host specific nor do any appear to cause disease. In my opinion there is no justification in this case for assigning different species names to this group of four organisms and I believe they should all be referred to as $E$. polecki, the name that has priority (Clark et al., 2006). Only people like myself who are interested in genetic diversity will care that four types exist and these can just as easily be identified as E. polecki types 1-4.

Precedent exists for this approach in the genus Entamoeba where significant genetic variation exists within other species, specifically E. coli, E. gingivalis and E. moshkouskii (Clark \& Diamond, 1997). Using SSU-rDNA restriction fragment length polymorphism analysis (riboprinting) several discrete variants were identified in each. Most variation has been identified in E. moshkovskii and it appears that additional sampling may show it to consist of a "bush" of variants rather than the few discrete branches defined initially (Silberman, personal communication). Again, based on SSU-rDNA sequence divergence, some of these variants differ by more than E. histolytica and E. dispar but in my opinion there is no justification for erecting new species. The same applies to the two genotypes of $D$. fragilis discussed earlier. However, it should be noted that these opinions are based on current knowledge and should our views of these organisms change so may the need for a new nomen- 
clature. Such was the case in Cryptosporidium where the two variants of $C$. parvum were shown to have distinct epidemiological and infection characteristics, as well as molecular divergence, and are now known as C. parvum and C. bominis (Morgan-Ryan et al., 2002). The last few years have seen a dramatic increase in our understanding of genetic variation in Blastocystis. In this parasite the existing nomenclature has proven to be problematic in several ways. Originally, all human infections were ascribed to B. hominis. Infections in other animals were mostly termed Blastocystis sp. but species names were erected for organisms in some hosts - for example B. ratti and B. galli from rats and chickens, respectively, as well as several from reptiles (Yoshikawa et al., 2004). However, extensive analysis of SSU-rDNA sequences by several groups has shown that the situation is complicated (Arisue et al., 2003; Abe, 2004; Yoshikawa et al., 2004; Noël et al., 2005; Menounos et al., 2007; Parkar et al., 2007; Stensvold et al., 2007b). Nine subtypes can be defined among mammal and bird isolates, all of which have been identified in humans. Nested within this variation are lineages with species from reptiles, which do not grow at $37^{\circ} \mathrm{C}$ and clearly represent distinct species. So the situation was taxonomically unworkable and could only be resolved by abandoning the use of species names for animal and bird isolates, including human isolates, and referring to them all as Blastocystis sp. (Stensvold et al., 2007a). Whether species names will eventually be assigned to each of the nine subtypes is unclear. Again the justification for this is questionable and at present the subtype identification system appears to be adequate. It is worth pointing out that the situation in Blastocystis only became clear after extensive sampling in many hosts and SSU-rDNA sequence analysis. The result has been a clear picture of variation in this genus and we can have confidence that the nine groups identified are stable. It is possible that additional minor subtypes may be uncovered but the taxonomy is flexible enough to incorporate them.

An overview of studies on these parasitic protists highlights the need for caution in several areas. Too much reliance on a single reference isolate without a survey of diversity can be misleading as important variation can be missed - indeed the reference isolate may prove to be atypical. Independent confirmation of "species-specific" PCR results should be considered when organisms are found in new or atypical hosts - the primer binding sites may be conserved but that need not be true of the rest of the gene. Morphological identification of parasites alone cannot be relied on for making epidemiological links. Extensive sampling coupled with sequence analysis may be required to obtain a clear picture of genetic variation in an organism - what appear to be discrete lineages may disappear when more data become available. Finally, I feel strongly that species names need to be useful and that this should be the primary motivation for any changes in nomenclature. They should clarify rather than obscure relationships.

After submission of this manuscript a third report of E. nuttalli was published - Suzuki J., Kobayashi S., Murata R., YanaGaWA Y. \& TAKeuchi T. Profiles of a pathogenic Entamoeba bistolytica-like variant with variations in the nucleotide sequence of the small subunit ribosomal RNA isolated from a primate (De Brazza's Guenon). Journal of Zoo Wildlife Medicine, 2007, 38, 471-474.

\section{REFERENCES}

ABE N. Molecular and phylogenetic analysis of Blastocystis isolates from various hosts. Vet. Parasitol., 2004, 120, 235242.

ANONYMOUS. WHO/PAHO/UNESCO report. A consultation with experts on amoebiasis. Epidemiol. Bull. PAHO, 1997a, 18, 13-14.

Anonymous. Entamoeba taxonomy. Bull. World Health Organ., 1997b, 75, 291-292.

Arisue N., Hashimoto T. \& Yoshikawa H. Sequence heterogeneity of the small subunit ribosomal RNA genes among Blastocystis isolates. Parasitology, 2003, 126, 1-9.

BALAMUTH W. Improved egg yolk medium for cultivation of Entamoeba histolytica and other intestinal protozoa. Am. J. Clin. Pathol., 1946, 16, 380-384.

Blessmann J., Van Linh P., Nu P.A., Thi H.D., Muller-MyHsoK B., Buss H. \& TANnich E. Epidemiology of amebiasis in a region of high incidence of amebic liver abscess in central Vietnam. Am.J. Trop. Med. Hyg., 2002, 66, 578-583.

Chan F.T., Guan M.X. \& Mackenzie A.M. Application of indirect immunofluorescence to detection of Dientamoeba fragilis trophozoites in fecal specimens. J. Clin. Microbiol., 1993, 31, 1710-1714.

CLARK C.G. \& Diamond L.S. Intraspecific variation and phylogenetic relationships in the genus Entamoeba as revealed by riboprinting. J. Euk. Microbiol., 1997, 44, 142-154.

Clark C.G. Cryptic genetic variation in parasitic protozoa. J. Med. Microbiol., 2000, 49, 489-491.

Clark C.G., Kaffashian F., Tawari B., Windsor J.J., TwiggFlesner A., Davies-Morel M.C.G., Blessmann J., Ebert F., PesChel B., Le Van A., Jackson C.J., Macfarlane L. \& Tannich E. New insights into the phylogeny of Entamoeba species provided by analysis of four new small-subunit rRNA genes. Int. J. Syst. Evol. Microbiol., 2006, 56, 2235-2239.

DiAmOND L.S. \& CLARK C.G. A redescription of Entamoeba histolytica Schaudinn, 1903 (Emended Walker, 1911) separating it from Entamoeba dispar Brumpt, 1925. J. Euk. Microbiol., 1993, 40, 340-344.

DWYER D.M. Analysis of the antigenic relationships among Trichomonas, Histomonas, Dientamoeba, and Entamoeba. I. Quantitative fluorescent antibody methods. J. Protozool., 1972a, 19, 316-325.

DWYer D.M. Analysis of the antigenic relationships among Trichomonas, Histomonas, Dientamoeba, and Entamoeba. II. Gel diffusion methods. J. Protozool., 1972b, 19, 326-332. 
DwYER D.M. Analysis of the antigenic relationships among Trichomonas, Histomonas, Dientamoeba, and Entamoeba. III. Immunoelectrophoresis technics. J. Protozool., 1974, 21, 139-145.

Hebert P.D., Cywinska A., Ball S.L. \& deWaard J.R. Biological identifications through DNA barcodes. Proc. Biol. Sci., 2003, 270, 313-321.

Johnson J.A. \& Clark C.G. Cryptic genetic diversity in Dientamoeba fragilis. J. Clin. Microbiol., 2000, 38, 4653-4654.

Menounos P.G., Spanakos G., Tegos N., Vassalos C.M., PapadoPOUlou C. \& VAKalis N.C. Direct detection of Blastocystis sp. in human faecal samples and subtype assignment using single strand conformational polymorphism and sequencing. Mol. Cell. Probes, 2007.

Morgan-Ryan U.M., Fall A., Ward L.A., Hijjawi N., Sulaiman I., Fayer R., Thompson R.C., Olson M., Lal A. \& Xiao L. Cryptosporidium hominis n. sp. (Apicomplexa: Cryptosporidiidae) from Homo sapiens. J. Eukaryot. Microbiol., 2002, 49, 433-440.

Noel C., Dufernez F., Gerbod D., Edgcomb V.P., DelgadoViscogliosi P., Ho L.C., Singh M., Wintjens R., Sogin M.L., Capron M., Pierce R., Zenner L. \& Viscogliosi E. Molecular phylogenies of Blastocystis isolates from different hosts: implications for genetic diversity, identification of species, and zoonosis. J. Clin. Microbiol., 2005, 43, 348-355.

Parkar U., Traub R.J., Kumar S., Mungthin M., Vitali S., Leelayoova S., Morris K. \& Thompson R.C. Direct characterization of Blastocystis from faeces by PCR and evidence of zoonotic potential. Parasitology, 2007, 134, 359-367.

Peek R., Reedeker F.R. \& van Gool T. Direct amplification and genotyping of Dientamoeba fragilis from human stool specimens. J. Clin. Microbiol., 2004, 42, 631-635.

Ponce Gordo F., Martínez Díaz R.A. \& Herrera S. Entamoeba struthionis n. sp. (Sarcomastigophora: Endamoebidae) from ostriches (Strutbio camelus). Vet. Parasitol., 2004, 119, 327335.

Sargeaunt P.G., Williams J.E. \& Grene J.D. The differentiation of invasive and non-invasive Entamoeba bistolytica by isoenzyme electrophoresis. Trans. R. Soc. Trop. Med. Hyg., 1978, 72, 519-521.

Silberman J.D., Clark C.G. \& Sogin M.L. Dientamoeba fragilis shares a recent common evolutionary history with the trichomonads. Mol. Biochem. Parasitol., 1996, 76, 311-314.

Stark D., Beebe N., Marriott D., Ellis J. \& Harkness J. Detection of Dientamoeba fragilis in fresh stool specimens using PCR. Int. J. Parasitol., 2005a, 35, 57-62.

Stark D., Beebe N., Marriott D., Ellis J. \& Harkness J. Prospective study of the prevalence, genotyping, and clinical relevance of Dientamoeba fragilis infections in an Australian population. J. Clin. Microbiol., 2005b, 43, 2718-2723.

Stensvold C.R., Suresh G.K., Tan K.S., Thompson R.C., Traub R.J., Viscogliosi E., Yoshikawa H. \& Clark C.G. Terminology for Blastocystis subtypes: a consensus. Trends Parasitol., 2007a, 23, 93-96.

Stensvold C.R., Traub R.J., von Samson-Himmelstjerna G., Jespersgaard C., Nielsen H.V. \& Thompson R.C. Blastocystis: subtyping isolates using pyrosequencing technology. Exp. Parasitol., 2007b, 116, 111-119.
Tachibana H., Yanagi T., Pandey K., Cheng X.J., Kobayashi S., Sherchand J.B. \& Kanbara H. An Entamoeba sp. strain isolated from rhesus monkey is virulent but genetically different from Entamoeba histolytica. Mol. Biochem. Parasitol., 2007, 153, 107-114.

Takano J., Narita T., Tachibana H., Terao K. \& Fujimoto K. Comparison of Entamoeba histolytica DNA isolated from a cynomolgus monkey with human isolates. Parasitol. Res., 2007, 101, 539-546.

Verweij J.J., Polderman A.M. \& Clark C.G. Genetic variation among human isolates of uninucleated cyst-producing Entamoeba species. J. Clin. Microbiol., 2001, 39, 1644-1646.

Windsor J.J., Clark C.G. \& Macfarlane L. Molecular typing of Dientamoeba fragilis. Br. J. Biomed. Sci., 2004, 61, 153155.

Yoshikawa H., Morimoto K., Wu Z., Singh M. \& Hashimoto T. Problems in speciation in the genus Blastocystis. Trends Parasitol., 2004, 20, 251-255. 